$$
\begin{aligned}
& \cot D G C=\cot \left(\alpha_{1}+\gamma_{2}\right)=\frac{\cot \alpha_{1} \cot \gamma_{2}-1}{\cot \alpha_{1}+\cot \gamma_{2}} \\
& =\frac{\left[\frac{m}{n}(\cot A+\cot C)+\cot A\right]\left[\frac{s}{r}(\cot C+\cot A)+\cot C\right]-1}{\frac{m}{n}(\cot A+\cot C)+\cot A+\frac{s}{r}(\cot C+\cot A)+\cot C} \\
& =\frac{\frac{m}{n} \cdot \frac{s}{r}(\cot A+\cot C)+\frac{s}{r} \cot A+\frac{m}{n} \cot C-\cot B}{\frac{m}{n}+\frac{s}{r}+1} \ldots \ldots . .
\end{aligned}
$$

3. If $A D, B E, C F$ are the medians of $\triangle A B C$,

(l) becomes $\cot \alpha_{1}=2 \cot A+\cot C$.

$\therefore \cot \alpha_{1}+\cot \beta_{1}+\cot \gamma_{1}=\cot \alpha_{2}+\cot \beta_{2}+\cot \gamma_{2}$

$$
=3(\cot A+\cot B+\cot C)=3 \cot \omega .
$$

(2) becomes $\cot \alpha_{1}-\cot \alpha_{2}=\cot C-\cot B$.

This may be proved as follows: Let $D N$ and $D L$ (Fig. 1) be $\perp^{r}$ to $C A$ and $A B$.

Then $A D^{2}-D C^{2}=A D^{2}-D B^{2} \quad \therefore A N^{2}-N C^{2}=A L^{2}-L B^{2}$.

$\therefore(A N-N C) A C=(A L-L B) A B$. But $\triangle A D C=\triangle A D B$.

$\therefore A B \cdot D L=A C \cdot D N$. Hence $\frac{A N-N C}{D N}=\frac{A L-L B}{D L}$.

$\therefore \cot \alpha_{1}-\cot C=\cot \alpha_{2}-\cot B$.

(3) becomes $\cot A D C=\frac{\cot B-\cot C}{2}=\frac{\cot \alpha_{2}-\cot \alpha_{1}}{2}$.

$\therefore \cot A D C+\cot B E A+\cot C F B=0$.

(4) becomes $\cot D G C=\frac{2 \cot A+2 \cot C-\cot B}{3}$.

$\therefore \cot D G C+\cot E G A+\cot F G B=\cot D G B+\cot E G C$

$+\cot F G A=\cot A+\cot B+\cot C=\cot \omega$, and $\cot D G C-\cot D G B=\cot C-\cot B=\cot \alpha_{1}-\cot \alpha_{2}=2 \cot A D B$.

Several of these results will be found in Hobson's "Trigonometry," Chap. XII.

A. G. Burgess.

\title{
An Area Proof of the Proposition
}

"If $A B$ is divided equally at $C$ and unequally at $D$, then $A D^{2}+D B^{2}=2 A C^{2}+2 C D^{2}$." 
For the figure shows that each side is made up of the whole area plus the square in the centre.

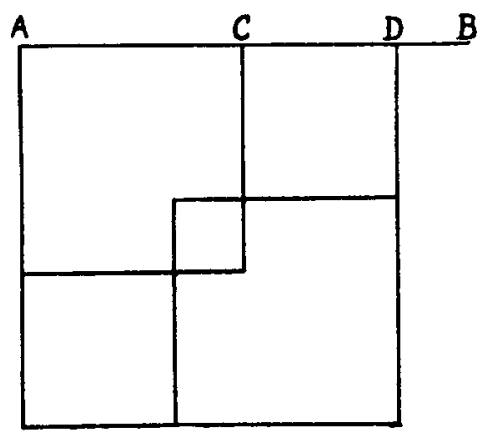

An exactly similar figure gives the case of external section at $D$.

[This note is taken from a letter dated 4th May 1916 from Professor R. J. T. Bell, who remarks "I got it in some L.C. papers and it was new to me and seemed very neat."-ED.]

\section{A Link Slide Rule for the Mechanical Solution of Quadratic Equations.}

[Note.-An earlier model of this slide rule was exhibited at a meeting of the Society in 1914. This note was held over during the war, and the instrument described now is an improved design.]

The instrument here described can be applied mechanically :

(a) to solve a numerical quadratic equation in the form

$$
x^{2}-a x+b=0 \text {. }
$$

(b) to evaluate $x^{2}-a x$ for assigned values of $x$ and $a$ and in particular to find its minimum value.

(c) to read the square or square root of a number.

(d) to multiply or divide two numbers.

(e) to evaluate the function $x+\frac{b}{x}$. 